\title{
Particle-level dynamics of clusters: Experiments in a gas-fluidized bed
}

\author{
Haifeng Wang ${ }^{1}$, Yanpei Chen ${ }^{1}$, and Wei Wang ${ }^{1}$ \\ ${ }^{1}$ Institute of Process Engineering Chinese Academy of Sciences
}

May 26, 2021

\begin{abstract}
The clustering is critical to understanding the multiscale behavior of fluidization. However, its time-resolved evolution on the particle level is seldom touched. Here, we explore both the time-averaged and time-resolved dynamics of clusters in a quasi-2D fluidized bed. Particle tracking velocimetry is adopted and then clusters are identified by using the Voronoi analysis. The time-averaged results show that the cluster hydrodynamic parameters depend highly on the cluster size and the distance from the wall. The number distribution of the cluster size follows a power law $\left({ }^{\sim} n_{c}{ }^{-2.2}\right)$ ) of the percolation theory except for large clusters $\left(n_{c}>100\right)$. The time-resolved analysis shows that the cluster coalescence can be simplified as a collision between two inelastic clusters, during which the net external force is roughly zero, and a snowplow model is proposed to predict its energy loss, $\Delta E \sim t^{3 / 2}$. The cluster rupture is suggested to be caused by increasing torque.
\end{abstract}

\section{Hosted file}

Particle-level dynamics of clusters-Experiments in a gas-fluidized bed.docx available at https://authorea.com/users/415950/articles/523703-particle-level-dynamics-of-clustersexperiments-in-a-gas-fluidized-bed 

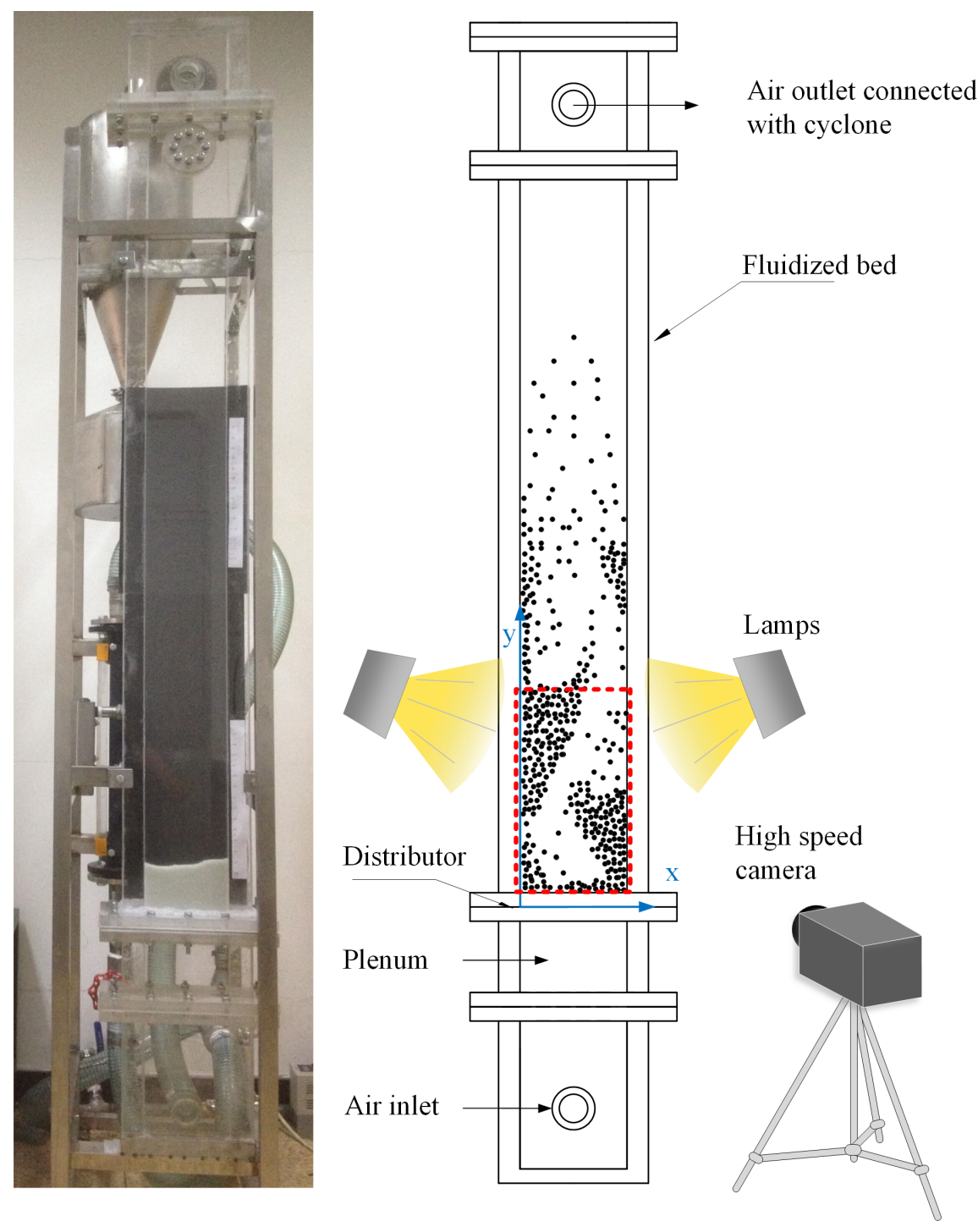


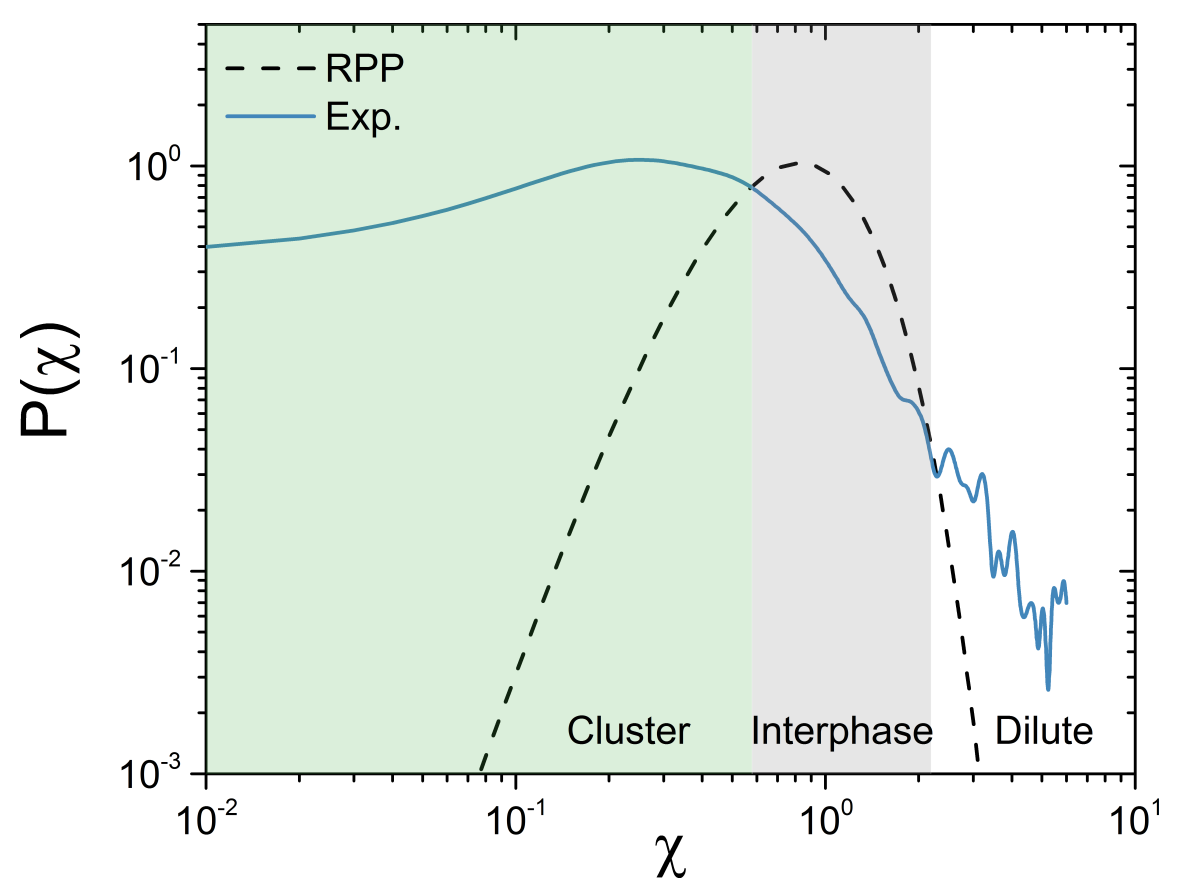

(a)

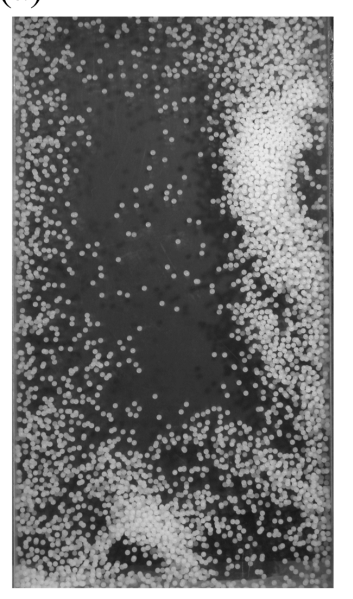

(b)

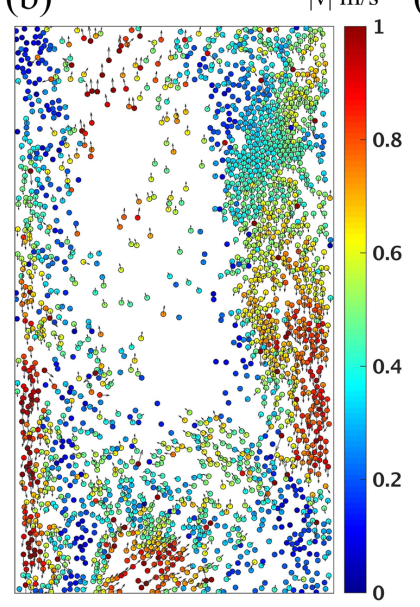

(c)

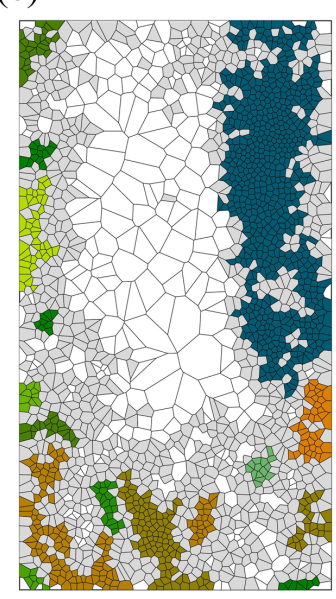

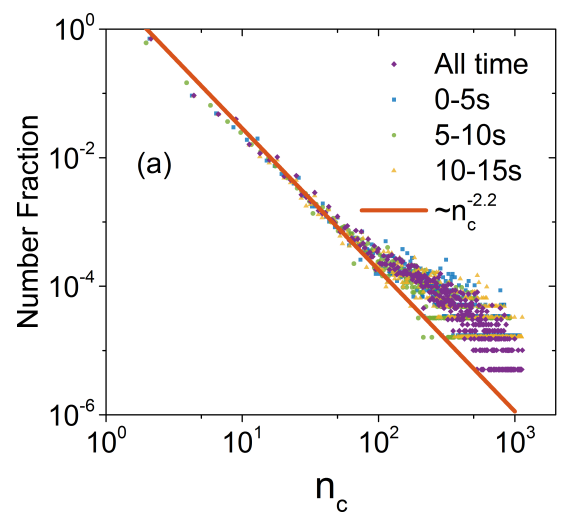

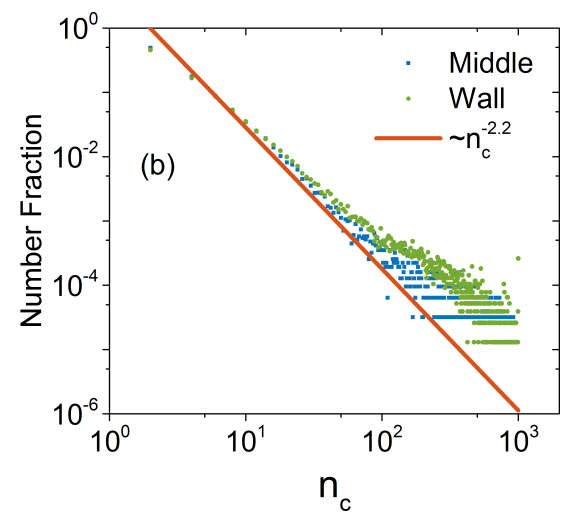



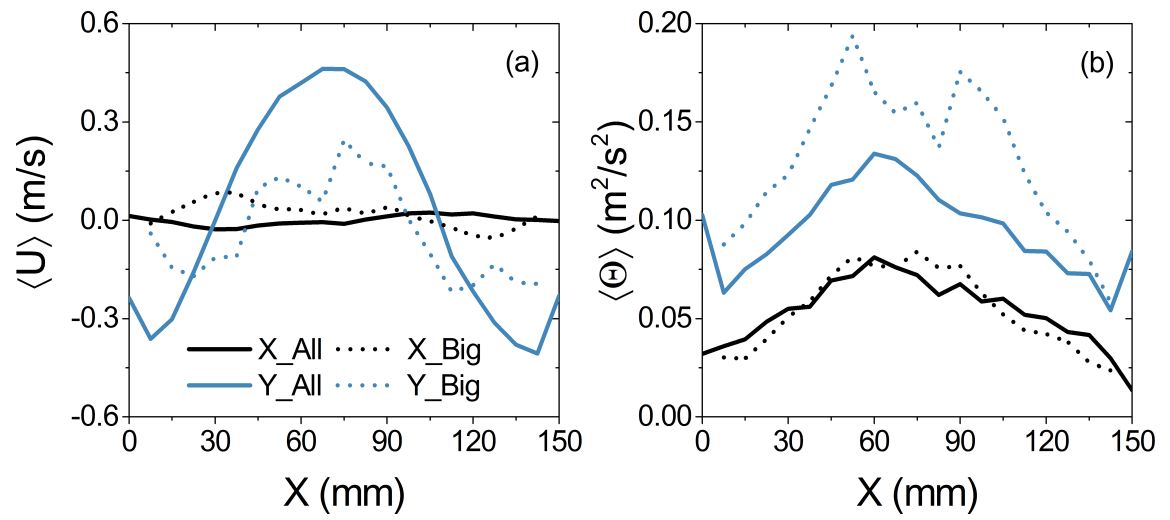

(a)

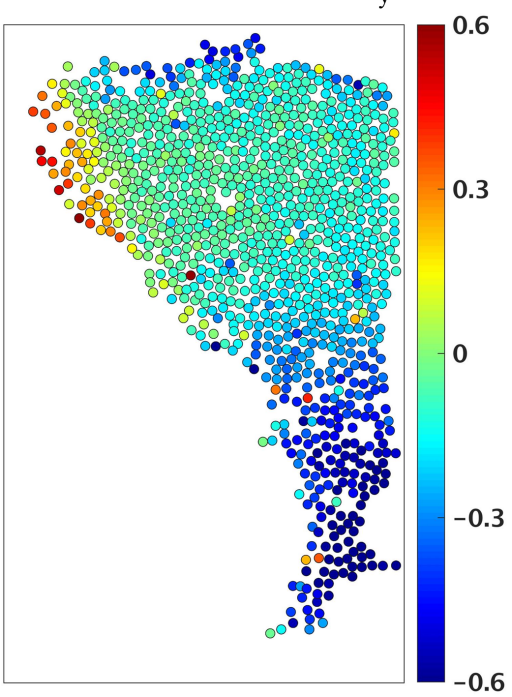

(b)

$\mathrm{a}_{\mathrm{y}} \mathrm{m} / \mathrm{s}^{2}$

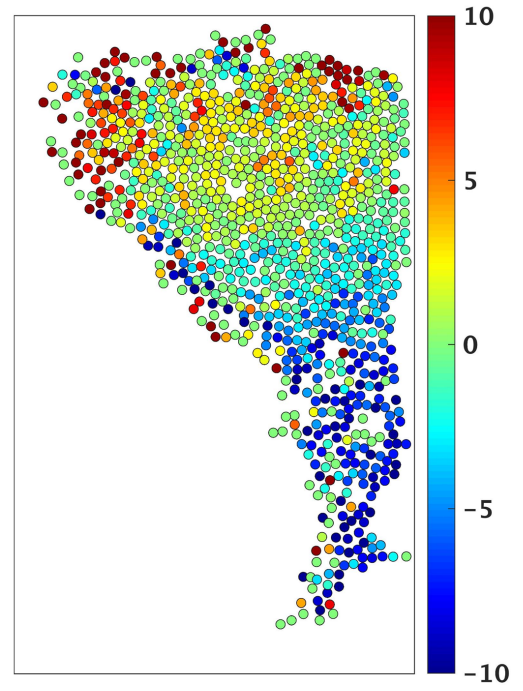



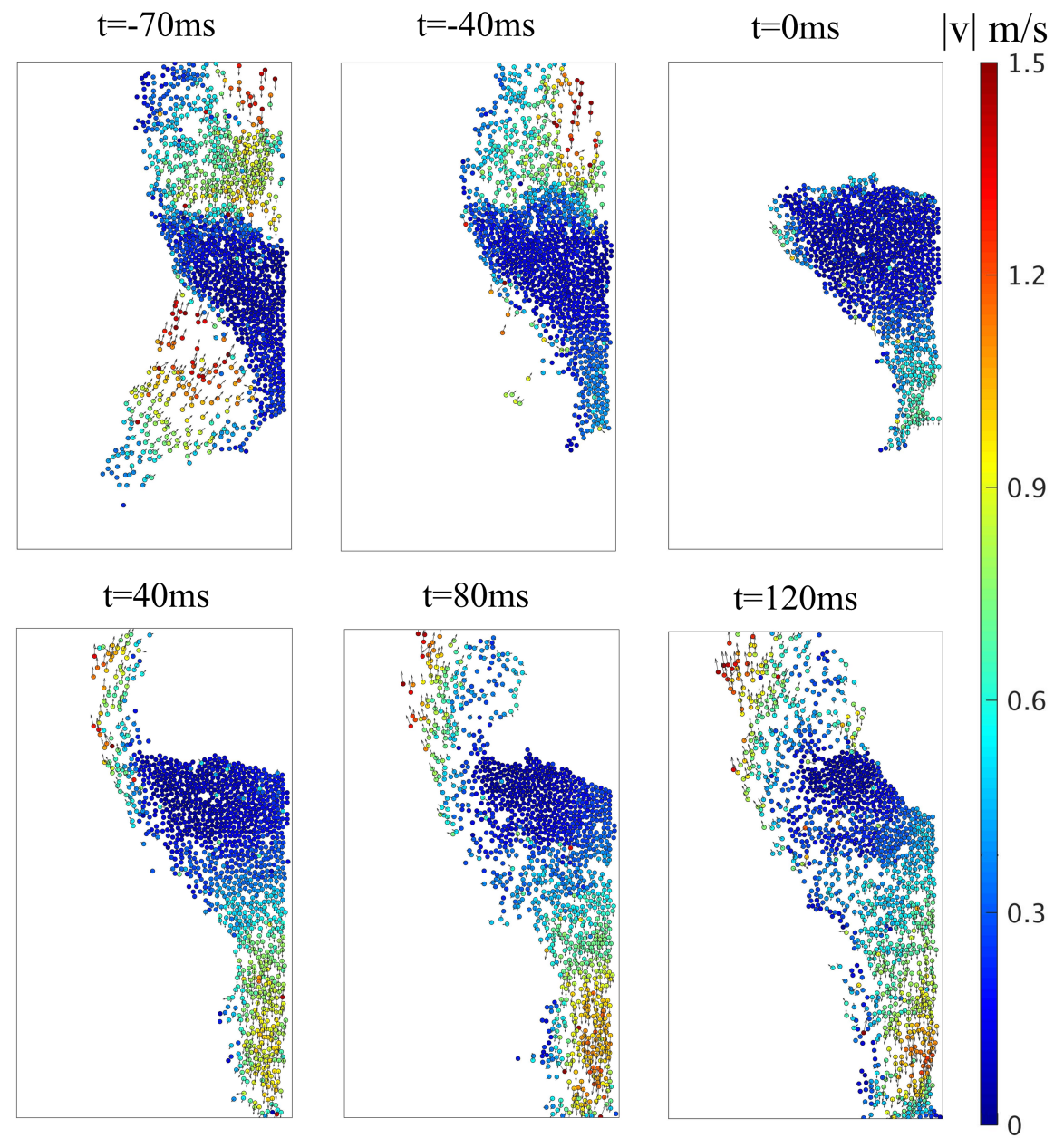


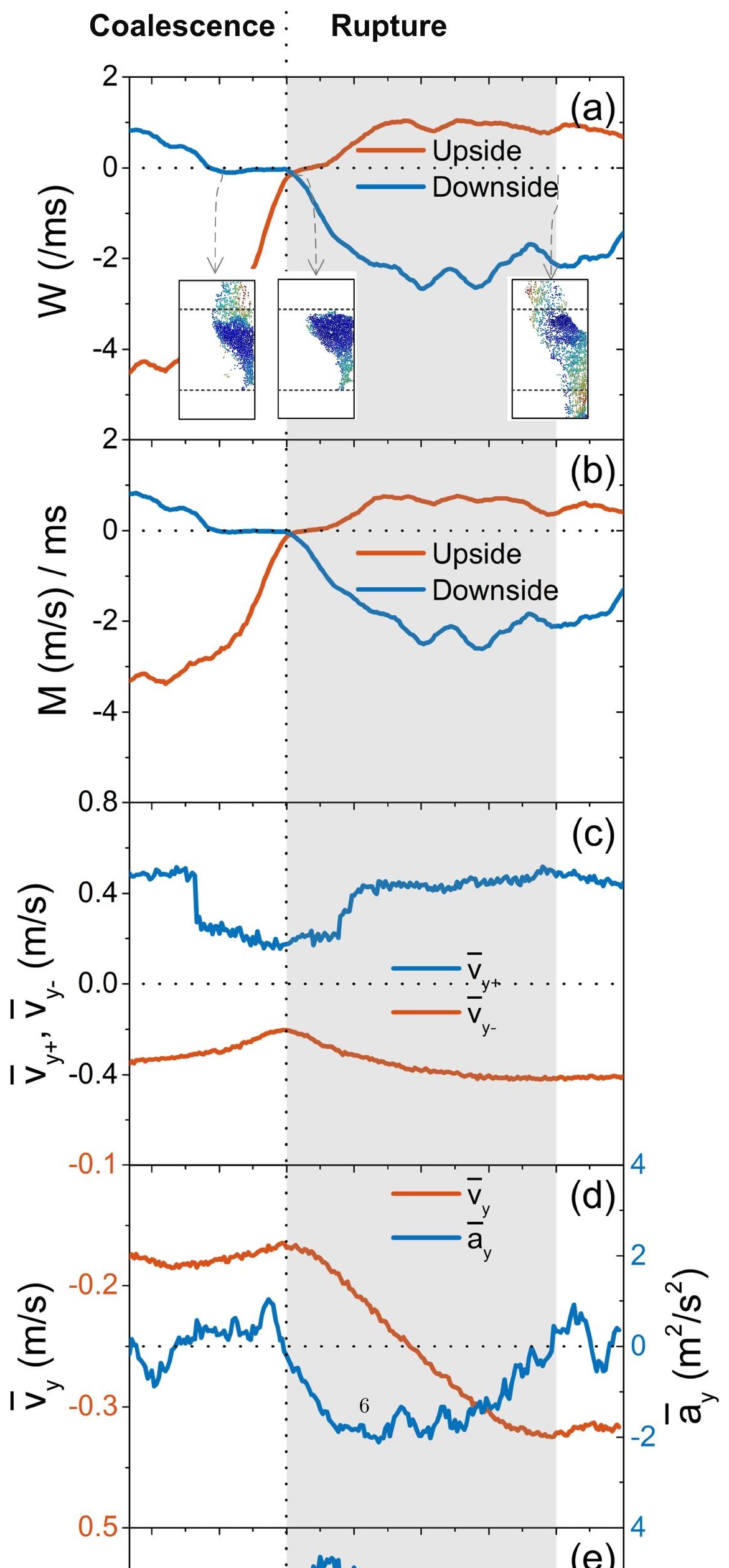


(a)
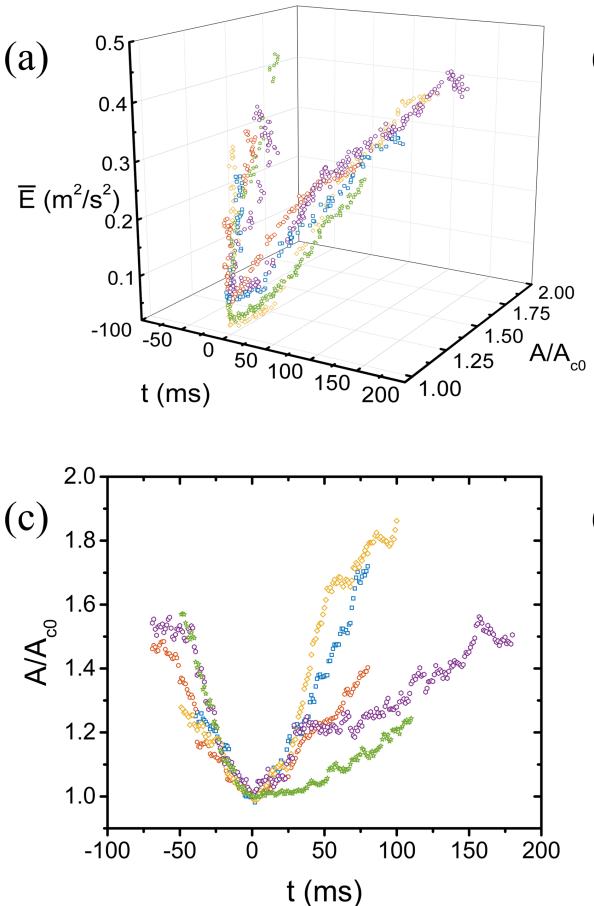
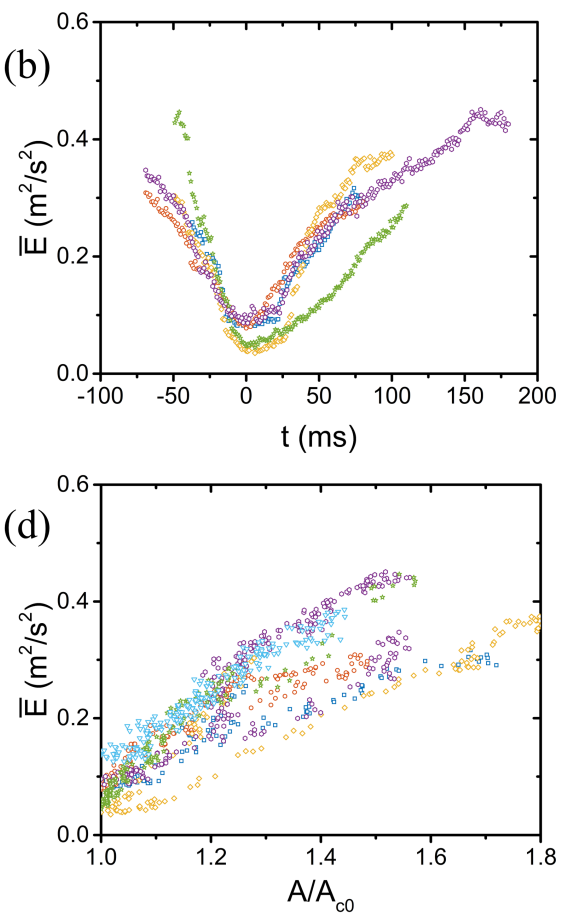

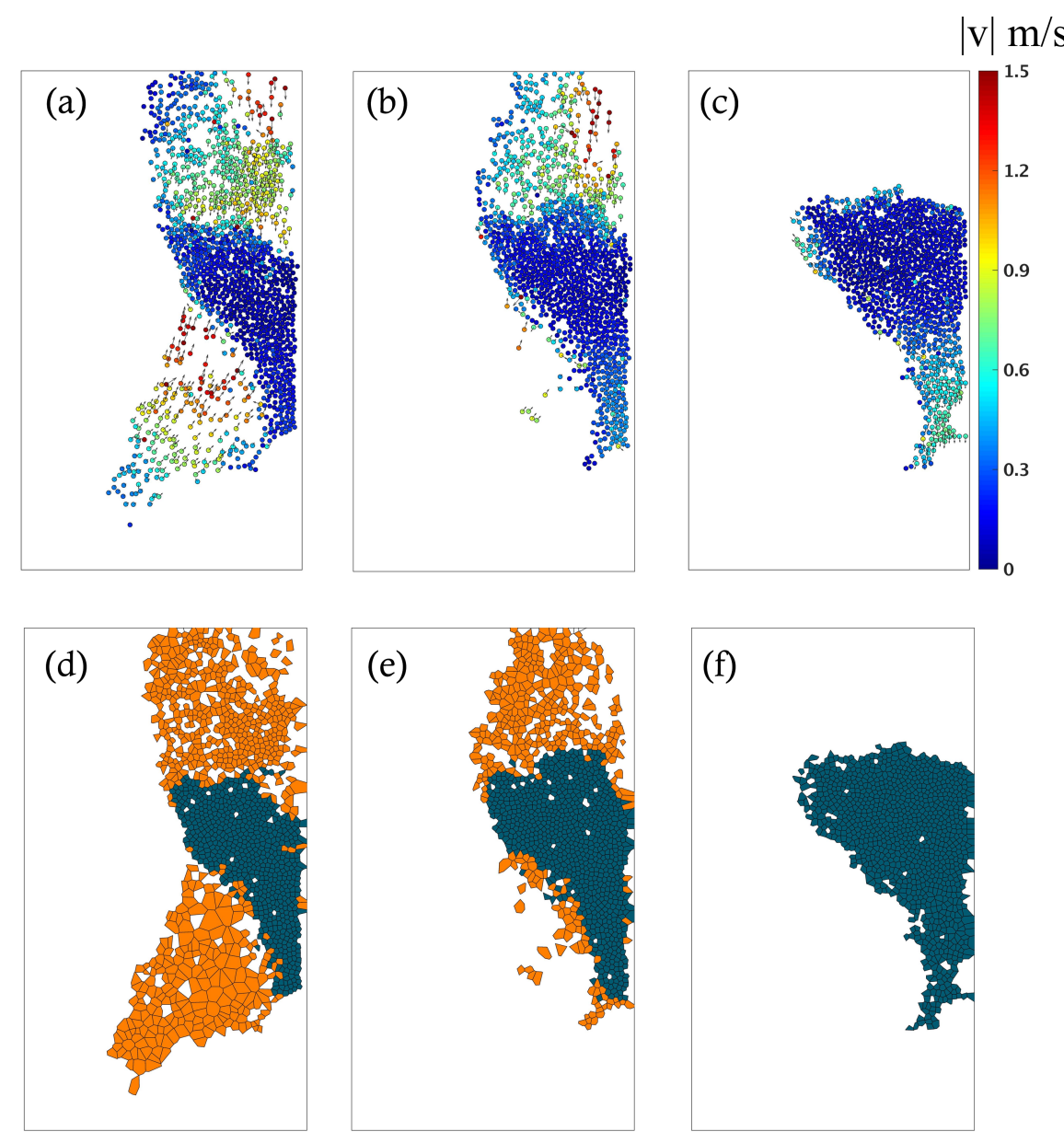

(a) Coalescence

(b) Cluster formation

(c) Rupture
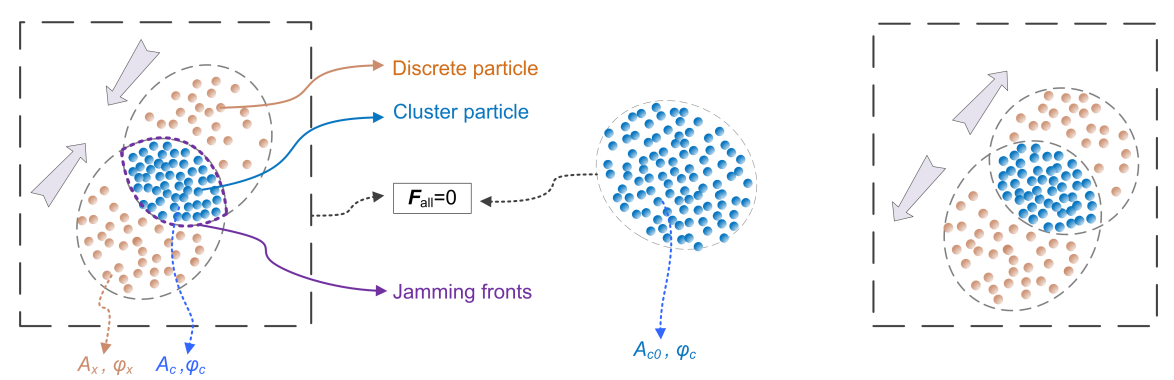

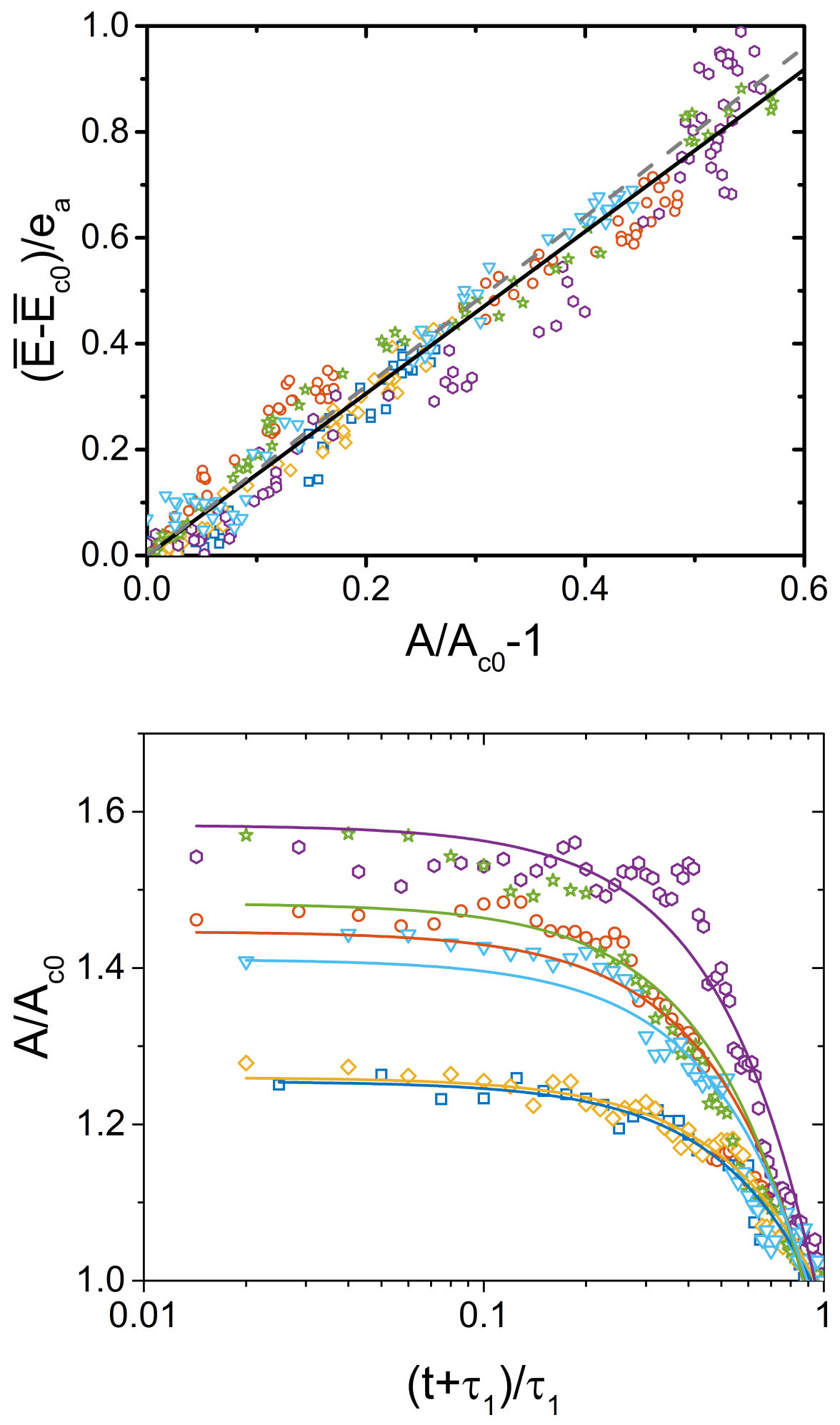


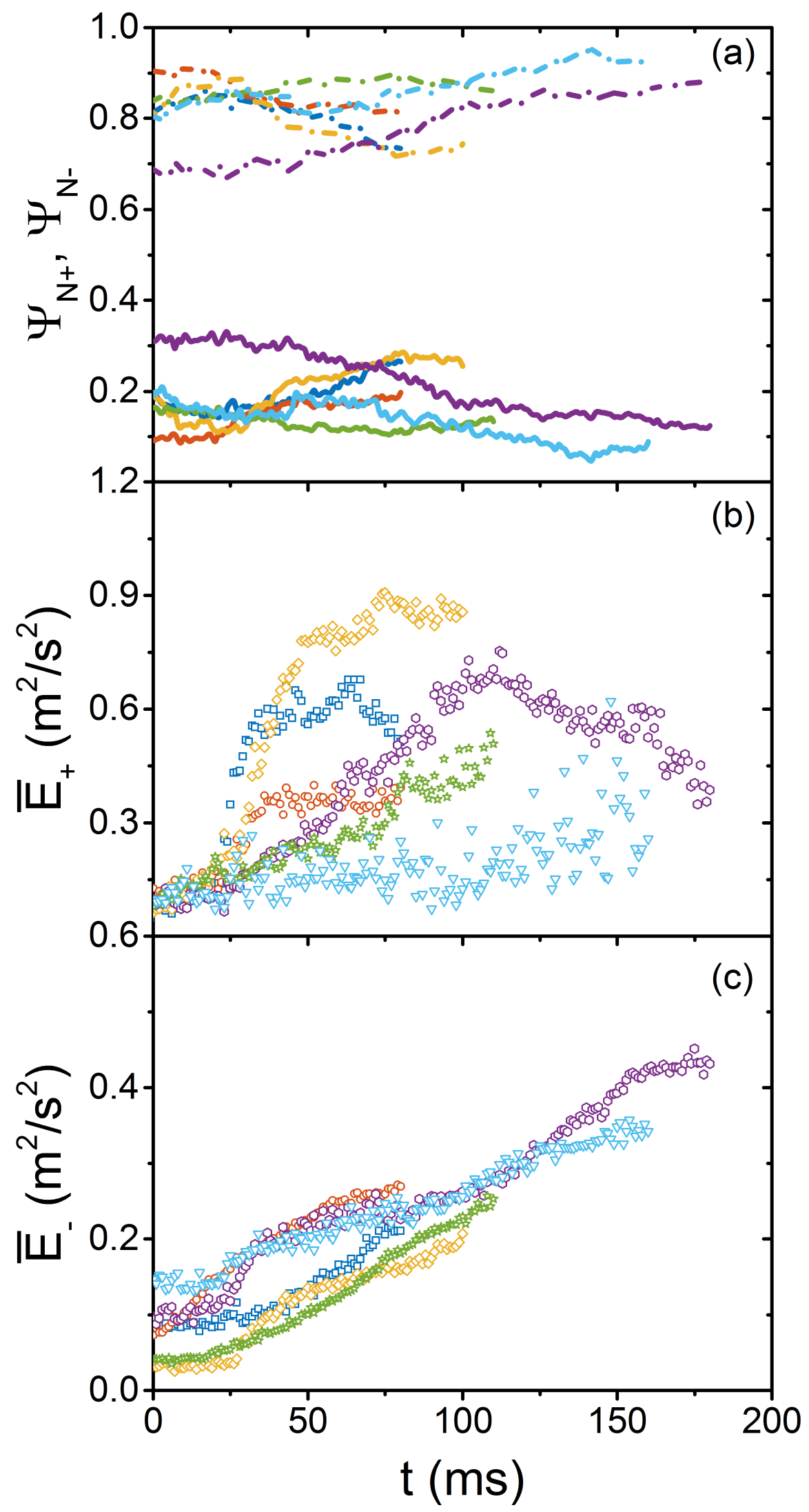



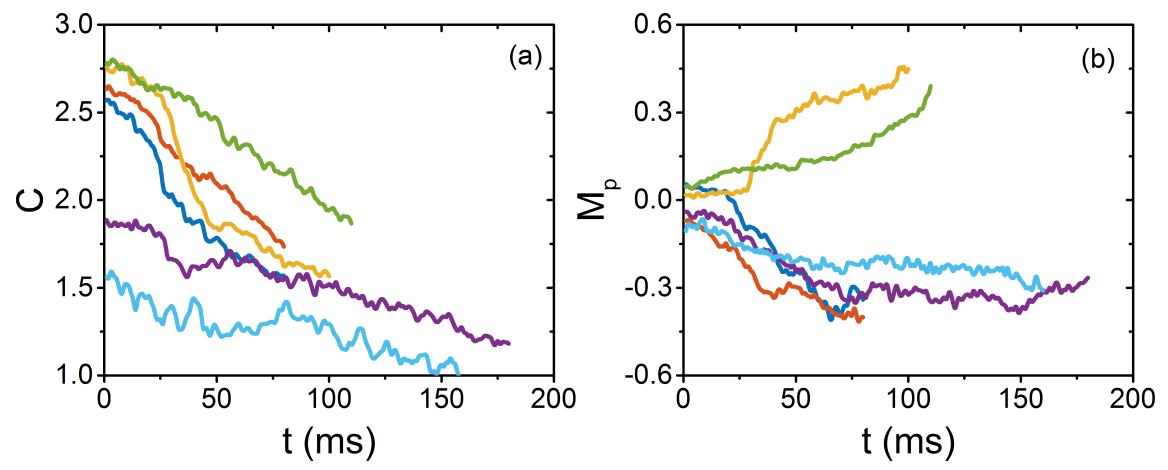